\title{
DETERMINAÇÃO DAS ISOTERMAS DE EQUILÍBRIO DAS SEMENTES DE UVA DAS VARIEDADES CABERNET SAUVIGNON E BORDÔ
}

\author{
Cissa Kelmer Bracht ${ }^{1}$ \\ Maraísa Lopes de Menezes ${ }^{1}$ \\ Miriam Carla Bonicontro Ambrosio Ugri ${ }^{1}$ \\ Nehemias Curvelo Pereira ${ }^{1}$
}

Resumo : O conhecimento da umidade de equilíbrio de uma matéria-prima é de grande importância para o processamento e controle das condições de armazenamento. As sementes de uva, que podem ser utilizadas para extração de óleo, apresentam uma elevada quantidade de umidade e durante a sua armazenagem podem sofrer mudanças físico-químicas e microbiológicas. Assim, esta pesquisa teve por objetivo determinar as isotermas de equilíbrio para as sementes de uva das variedades Cabernet Sauvignon e Bordô, visando à obtenção de dados que indique uma ${ }^{1}$ melhor condição de armazenamento e processamento desta matéria-prima. As isotermas de equilíbrio foram determinadas nas temperaturas de 25,35 e $50{ }^{\circ} \mathrm{C}$ utilizando o método estático. Foram ajustados aos dados experimentais os modelos de Henderson, Henderson Modificado, Motta Lima, BET, Sabbah e Oswin, utilizando o software Statistica $7.1^{\circledR}$. Com base nos coeficientes de determinação $\left(\mathrm{R}^{2}\right)$, nos valores do teste $\mathrm{F}$ e da raiz quadrada do erro médio (RM), foi possível determinar o modelo que melhor se ajustou aos dados experimentais. Para a determinação do calor de sorção, foi utilizada a equação de Clausius-Clapeyron, a atividade de água foi estimada pelo modelo de Henderson (1952) para as temperaturas de 25,35 e $50{ }^{\circ} \mathrm{C}$ e as umidades de equilíbrio variaram de 2 a $15 \%$. Os resultados mostraram que a umidade relativa do ambiente em que as sementes são armazenadas não poderá ser maior que $75 \%$ para que as mesmas permaneçam com um conteúdo de umidade menor que $10 \%$ (b.s.) até o seu processamento. Verificou-se, também, que os modelos que melhor se ajustaram aos dados experimentais obtidos para as isotermas de equilíbrio foram os de Henderson e Henderson Modificado, em todas as condições estudadas. Com relação ao calor de sorção, foi verificado que este diminuiu com o aumento da umidade de equilíbrio, com valores variando entre $2329 \mathrm{~kJ} / \mathrm{kg}$ e $3403 \mathrm{~kJ} / \mathrm{kg}$ para as sementes de uva da variedade Cabernet Sauvignon e entre 2225 e 4077 kJ/kg para as sementes de Bordô.

Palavras-chave: umidade de equilíbrio, isotermas, ajuste de modelos matemáticos, calor de sorção.

Abstract : The knowledge of the equilibrium moisture content of a raw material is very important for its processing and for controlling the storage conditions. Grape seeds, which could be used for oil extraction, have high moisture content and during storage, they are subject to physicochemical and microbiologic changes. Thus, this study aimed to determine the equilibrium isotherms for seeds of grape varieties, Cabernet Sauvignon and Ives, with the intention to have information to improve the storage and processing conditions of this raw material. The equilibrium isotherms were determined at 25, 35 and $50{ }^{\circ} \mathrm{C}$ by static method. The models Henderson, Modified Henderson, Motta Lima, BET, Oswin and Sabbah were adjusted to experimental data using the software Statistica 7.1 ®. Based on the coefficients of determination $\left(\mathrm{R}^{2}\right)$, the values of the $\mathrm{F}$ test and the root mean square error (RM), it was possible to verify the model that best adjust to experimental data. To determine the heat of desorption, the Clausius-Clapeyron equation was used, and the

\footnotetext{
${ }^{1}$ Programa de Pós-graduação Stricto Sensu em Engenharia Química da Universidade Estadual de Maringá UEM. Email: cissaum@hotmail.com
} 
water activity was estimated by the Henderson model (1952) at temperatures of 25, 35 and $50{ }^{\circ} \mathrm{C}$. The equilibrium moisture content chosen were 2 to $15 \%$. The results showed that the relative humidity of the environment in which the seeds are stored cannot be more than $75 \%$ to allow them remain with moisture content below $10 \%(\mathrm{db})$. It was verified also that the models that best adjust to experimental data of equilibrium isotherms were Henderson and Modified Henderson in all studied conditions. Concerning the heat of desorption, it was verified that it decreases with an increase in moisture, and the founded values were between $2329 \mathrm{~kJ} / \mathrm{kg}$ and $3403 \mathrm{~kJ} / \mathrm{kg}$ for Cabernet seeds and between 2225 and $4077 \mathrm{~kJ} / \mathrm{kg}$ for Ives seeds.

Keywords: equilibrium moisture content, equilibrium isotherms, mathematical models adjustment, heat of desorption.

\section{INTRODUÇÃO}

O Brasil, com sua economia fortemente baseada na agricultura, contribui para a geração de uma grande quantidade de resíduos resultantes das atividades de processamento.

$\mathrm{O}$ tratamento de subprodutos agrícolas vem recebendo mais atenção nos últimos anos, tendo em vista o seu aproveitamento, a despoluição do ambiente e, sempre que possível, com ambas as finalidades.

A indústria vinícola produz grandes quantidades de resíduos que ainda são muito pouco utilizados. Os resíduos de maior impacto são os sedimentos da fermentação do vinho e o bagaço de uva, sendo que boa parte deste é composto de sementes da fruta ( $\mathrm{S}^{\prime}$ enyi, 2006).

Os resíduos do processamento da uva contêm muitas substâncias de interesse comercial. Das sementes podem ser extraídas substâncias como proteínas, taninos e o óleo, que é considerado o composto mais valioso devido ao rico conteúdo de ácidos graxos, vitamina E, além de outras características peculiares, podendo ser utilizado nas indústrias química, alimentícia e farmacêutica (Miguel, 1983).

Existem diversas técnicas que podem ser aplicadas para a extração deste óleo, sendo que as mais utilizadas são: a extração por solvente e a extração por prensagem. Em ambos os casos, a umidade presente na semente dificulta o processo de extração. No caso da extração por prensagem, a umidade requerida é de $10 \%$, em base seca
(Roberts et al., 2008).

A armazenagem é um ponto crucial para a preservação da boa qualidade da matéria-prima, sendo que o controle adequado de temperatura e umidade do ambiente proporciona um maior tempo de armazenamento, reduzindo o desenvolvimento microbiológico.

A umidade de equilíbrio é definida como a menor umidade que um material pode atingir em um processo de secagem sob condições constantes de pressão e temperatura. Esta pode variar em função da estrutura do material sólido, da umidade e da temperatura do ar de secagem (Foust et al., 1982).

A determinação da umidade de equilíbrio estabelece parâmetros (temperatura e umidade relativa) que norteiam o valor do conteúdo de umidade em que materiais sólidos podem ser secos ou armazenados seguramente (Labuza, 1968).

Segundo Brooker et al. (1992), a umidade de equilíbrio em sementes ricas em óleo é mais baixa que em sementes amiláceas, quando armazenadas em condições ambientais semelhantes, pois absorvem menos água por serem hidrófobas.

O conhecimento das isotermas de equilíbrio do sólido objeto de estudo é de grande importância na área da tecnologia e da ciência dos materiais para a resolução de muitos problemas, tais como: avaliação e caracterização das ligações da água, análise do calor de sorção, otimização do processo, avaliação dos problemas de estocagem e embalagem e, também, para a 
estimativa da estabilidade química, física e microbiológica de materiais higroscópicos (Ditchfield, 2000).

A técnica estática para a determinação das isotermas de equilíbrio constitui em um método simples, tendo como vantagem a facilidade de se atingir as condições termodinâmicas constantes em recipientes de pequeno volume, sendo, portanto, a mais utilizada. A desvantagem deste método está no tempo gasto e no custo, que depende da solução indicada para o conhecimento da umidade relativa do ar a uma determinada temperatura (Barrozo et al., 2000).

As isotermas de equilíbrio apresentam diferentes formas. Brunauer et al. (1938) classificaram as isotermas em cinco tipos. As isotermas do tipo I e do tipo II são chamadas isotermas de Langmuir e sigmoidal ou de estrutura em S, respectivamente. Nenhum nome específico foi dado para os demais tipos de isotermas de equilíbrio. Os tipos II e III têm uma estreita relação com os tipos IV e $\mathrm{V}$, com a exceção que a máxima adsorção ocorre em pressões abaixo da pressão de vapor de água do meio.

As isotermas de forma sigmoidal (tipo II) são características de muitos materiais proteicos ou farináceos (farinha de trigo), amidos e amidos modificados (Ascheri et al., 2005). No entanto, alimentos como frutas, produtos de confeitaria e café solúvel, com elevado teor de açúcar e outras moléculas solúveis de baixo peso molecular e pobre em polímeros de alto peso molecular, exibem isotermas de equilíbrio do tipo I (Chetana et al., 2005).

Vários são os modelos empíricos, semi-empíricos ou teóricos que podem ser utilizados para descrever matematicamente as isotermas de equilíbrio. Dentre as diversas equações utilizadas para expressar o teor de umidade de equilíbrio de sementes, em função da temperatura e da umidade relativa ou atividade de água, destacamse as de Henderson, Henderson Modificado, Sabbah, Oswin, entre outros, pela sua precisão e generalidade no uso (Chen e Jayas, 1998).
A equação de Henderson Modificado é tida pela Sociedade Americana de Engenheiros Agrícolas (ASAE, 1993) como uma das mais utilizadas em produtos agrícolas.

A relação entre o conteúdo de umidade do produto analisado $\left(\mathrm{X}_{\mathrm{e}}\right)$, a atividade de água $\left(\mathrm{a}_{\mathrm{w}}\right)$ e a temperatura, fornece informações importantes com respeito ao fenômeno de sorção, tais como: a água absorvida na monocamada molecular ou o calor de sorção para as camadas subsequentes (Mesquita et al., 2001).

Segundo Yoshida (1997), o calor de sorção é, geralmente, obtido a partir de dados de sorção e é definido como a diferença entre a entalpia da água na fase vapor e a entalpia da água líquida adsorvida no sólido a uma dada concentração, isto é, ele representa a quantidade de energia necessária para evaporar a água adsorvida, na fase sólida. Seu valor é determinado pela Equação de Clausius-Clapeyron, que relaciona a mudança da atividade de água com a temperatura.

Com o intuito de amenizar as alterações físicas, químicas $\mathrm{e}$ microbiológicas que podem ocorrer durante o armazenamento de produtos agrícolas, como as sementes de uva, se faz necessário o conhecimento das relações existentes entre o produto, a temperatura e a atividade de água, sendo este, o objetivo deste estudo.

\section{MATERIAL E MÉTODOS}

\subsection{MATERIAL}

As sementes de uva da variedade Cabernet Sauvignon foram adquiridas de fornecedores da cidade de Toledo-PR e as sementes de uva da variedade Bordô foram adquiridas de fornecedores da cidade de Pinheiro Preto-SC.

As sementes passaram por um processo de limpeza no qual foram colocadas em peneiras e lavadas em água corrente para a retirada das cascas, engaço e açúcar. Em seguida, foram lavadas novamente com água deionizada e, posteriormente, com água deionizada 
aquecida a $100^{\circ} \mathrm{C}$, conforme Freitas (2007). As sementes limpas foram secas à temperatura ambiente, embaladas em sacos plásticos e guardadas sob refrigeração $\left(-15^{\circ} \mathrm{C}\right)$ até $\mathrm{o}$ momento de uso.

\subsection{DETERMINAÇÃO DAS ISOTERMAS DE EQUILÍBRIO}

As isotermas de equilíbrio foram determinadas para as sementes de uva das variedades Cabernet Sauvignon e Bordô nas temperaturas de 25,35 e $50{ }^{\circ} \mathrm{C}$.

Para a obtenção dos dados de equilíbrio, foi utilizado o método estático em estufa, sendo utilizadas soluções saturadas de oito tipos de sais: cloreto de lítio $(\mathrm{LiCl})$, acetato de potássio $\left(\mathrm{CH}_{3} \mathrm{CO}_{2} \mathrm{~K}\right)$, cloreto de magnésio
$\left(\mathrm{MgCl}_{2} \cdot 6 \mathrm{H}_{2} \mathrm{O}\right)$, carbonato de potássio $\left(\mathrm{K}_{2} \mathrm{CO}_{3}\right)$, nitrato de magnésio $\left(\mathrm{Mg}\left(\mathrm{NO}_{3}\right)\right.$ 2), nitrito de sódio $\left(\mathrm{NaNO}_{2}\right)$, cloreto de sódio $(\mathrm{NaCl})$ e cloreto de potássio $(\mathrm{KCl})$.

A preparação das soluções salinas saturadas requer cuidados especiais, principalmente quando o sal for hexahidratado, como é o caso do $\mathrm{MgCl}_{2} \cdot 6 \mathrm{H}_{2} \mathrm{O}$ e $\mathrm{Mg}\left(\mathrm{NO}_{3}\right)_{2} \cdot 6 \mathrm{H}_{2} \mathrm{O}$. Tais soluções liberam moléculas de água que, em contato com a umidade, diluem a solução preparada, modificando seu estado de saturação e, portanto, a umidade relativa desejada (Sousa, 2003; Fiorentin et al., 2010). As umidades relativas de cada uma das soluções saturadas dos sais em relação à temperatura estão apresentadas na Tabela 1 .

Tabela 1. Umidade relativa dos sais em relação à temperatura

\begin{tabular}{c|c|c|c|c|c|c|c|c}
\hline \multicolumn{8}{c}{ Sal/Umidade Relativa } \\
\hline $\begin{array}{c}\mathrm{T} \\
\left({ }^{\circ} \mathrm{C}\right)\end{array}$ & $\mathrm{LiCl}$ & $\mathrm{CH}_{3} \mathrm{CO}_{2} \mathrm{~K}$ & $\begin{array}{c}\mathrm{MgCl}_{2} \cdot \\
6 \mathrm{H}_{2} \mathrm{O}\end{array}$ & $\mathrm{K}_{2} \mathrm{CO}_{3}$ & $\begin{array}{c}\mathrm{Mg} \\
\left(\mathrm{NO}_{3}\right)_{2}\end{array}$ & $\mathrm{NaNO}_{2}$ & $\mathrm{NaCl}$ & $\mathrm{KCl}$ \\
\hline 25 & 0,113 & 0,225 & 0,328 & 0,432 & 0,511 & 0,645 & 0,753 & 0,843 \\
\hline 35 & 0,112 & 0,208 & 0,320 & 0,432 & 0,548 & 0,621 & 0,749 & 0,822 \\
\hline 50 & 0,111 & 0,192 & 0,312 & 0,433 & 0,585 & 0,597 & 0,746 & 0,802 \\
\hline
\end{tabular}

Fonte: Perry e Chilton (1980)

As soluções de todos os sais foram preparadas colocando-se $50 \mathrm{ml}$ de água destilada dentro de cada um dos frascos, com exceção dos sais hidratados, cuja quantidade de água destilada utilizada foi de $25 \mathrm{ml}$, uma vez que estes dobram de volume em contato com a água.

As amostras de semente foram primeiramente secas em estufa a $105^{\circ} \mathrm{C}$, por 24 horas. Em seguida, foram pesadas em balança analítica (precisão $\pm 0,0001$ g) para a determinação da massa seca.

As amostras de semente secas foram colocadas em recipientes plásticos vazados, sendo o conjunto pesado em balança analítica (precisão $\pm 0,0001 \mathrm{~g}$ ).

Em seguida, o recipiente plástico com a amostra foi colocado sobre um suporte que se encontrava no interior do frasco de vidro, o qual foi fechado e colocado na estufa na temperatura prédeterminada, conforme mostra a Figura 1.

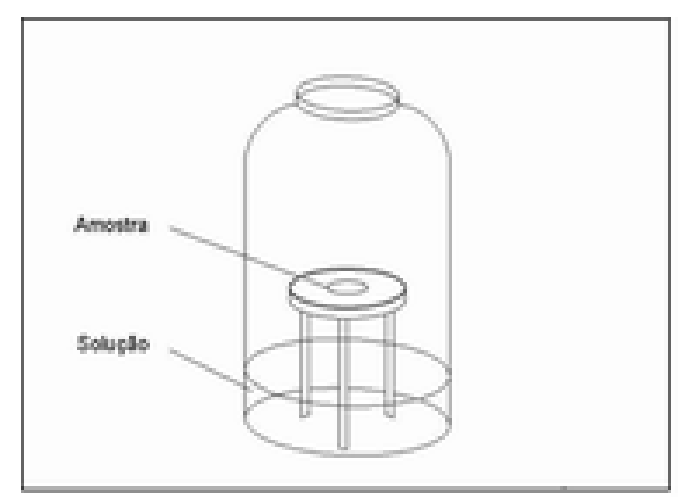

Figura 1: Frasco contendo a solução salina e a amostra de semente de uva

Em intervalos de tempo de 48 horas, foram realizadas pesagens do recipiente plástico com a amostra, sendo este procedimento repetido até que se tivessem três leituras de massa constante.

Utilizando a Equação 1, foram calculadas as umidades de equilíbrio $\left(\mathrm{X}_{\mathrm{e}}\right)$ das amostras para cada sal. Com estes resultados, foram traçadas as isotermas de 
equilíbrio, $X_{e}$ (umidade de equilíbrio) em função de $\mathrm{a}_{\mathrm{w}}$ (atividade de água), para cada uma das três temperaturas testadas.

$$
X(\text { b.s. })=\frac{M_{\text {umida }}-M_{\mathrm{sec} a}}{M_{\mathrm{sec} a}}
$$

Em que: $\mathrm{X}_{\mathrm{e}}$ (b.s) é a razão entre a massa úmida e a massa seca de sólido, em um determinado tempo, em base seca (b.s.); $\mathrm{M}_{\text {úmida }}$ é a massa de água presente na amostra (g) e $M_{\text {seca }}$ a massa seca obtida em estufa $(\mathrm{g})$.

\subsection{MODELAGEM ISOTERMAS DE EQUILÍBRIO}

DAS

Vários modelos têm sido propostos na literatura abordando as isotermas de equilíbrio. A Tabela 2 apresenta alguns destes modelos, sendo estes utilizados para o ajuste aos dados experimentais.

Tabela 2. Modelos de sorção empregados na modelagem das isotermas de equilíbrio

\begin{tabular}{c|c|c}
\hline Modelo & Equação & Referência \\
\hline $\begin{array}{c}\text { Henderson } \\
(1952)\end{array}$ & $\mathrm{Xe}=\mathrm{D}\left(\frac{1}{\mathrm{~T}} \ln \left(\frac{1}{1-\mathrm{a}_{\mathrm{w}}}\right)\right)^{\mathrm{B}}$ & $\begin{array}{c}\text { Henderson } \\
(1952)\end{array}$ \\
\hline $\begin{array}{c}\text { Henderson } \\
\text { modificado } \\
(1968)\end{array}$ & $\mathrm{Xe}=\left(\frac{-\ln \left(1-\mathrm{a}_{\mathrm{w}}\right)}{\mathrm{D}(\mathrm{T}+\mathrm{B})}\right)^{1 / \mathrm{C}}$ & $\begin{array}{c}\text { Thompson } \\
(1968)\end{array}$ \\
\hline $\begin{array}{c}\text { Motta Lima } \\
(1999)\end{array}$ & $\mathrm{Xe}=\frac{\ln \left(1-\mathrm{a}_{\mathrm{w}}\right)^{\mathrm{C}}}{\mathrm{D} \exp (-\mathrm{B} / \mathrm{T})}$ & $\begin{array}{c}\text { Motta Lima } \\
(1999)\end{array}$ \\
\hline $\begin{array}{c}\text { BET } \\
(1938)\end{array}$ & $\mathrm{Xe}=\frac{\mathrm{X} \mathrm{m} \mathrm{Ca}_{\mathrm{w}}}{\left(1-\mathrm{a}_{\mathrm{w}}\right)\left(1+\mathrm{Ca}_{\mathrm{w}}-\mathrm{a}_{\mathrm{w}}\right)}$ & $\begin{array}{c}\text { Chirife } \mathrm{e} \\
\text { Iglesias } \\
(1978)\end{array}$ \\
\hline $\begin{array}{c}\text { Sabbah } \\
(2001)\end{array}$ & $\mathrm{Xe}=\mathrm{K} \frac{\mathrm{a}_{\mathrm{w}}^{\mathrm{B}}}{\mathrm{T}}$ & $\begin{array}{c}\text { Mesquita et } \\
\text { al. }, \\
(2001)\end{array}$ \\
\hline $\begin{array}{c}\text { Oswin } \\
(1946)\end{array}$ & $\mathrm{Xe}=\mathrm{m}\left(\frac{\mathrm{a}_{\mathrm{w}}}{1-\mathrm{a}_{\mathrm{w}}}\right)^{\mathrm{n}}$ & $\begin{array}{c}\text { Oswin } \\
(1946)\end{array}$ \\
\hline
\end{tabular}

Em que: $X_{m}$ é o conteúdo de umidade na monocamada molecular $(\mathrm{g} / \mathrm{g}), \mathrm{a}_{\mathrm{w}}$ é a atividade de água (varia de 0 a 1) e B, $\mathrm{C}$ e D são constantes dos modelos que dependem da natureza do produto.

As constantes dos modelos foram obtidas por regressão não linear (método Quasi-Newton), com o auxílio do software Statistica $7.1{ }^{\circledR}$.

\subsection{ANÁLISE ESTATÍSTICA}

Os melhores modelos foram escolhidos com base nos maiores valores do coeficiente de determinação $\left(\mathrm{R}^{2}\right)$ e do Teste $\mathrm{F}$ e nos menores valores da raiz do erro médio (RM). Os valores para o Teste $\mathrm{F}$ e para RM foram encontrados utilizando as Equações 2 e 3.

$$
\begin{gathered}
\mathrm{RM}=\left[\frac{1}{\mathrm{~N}} \sum_{\mathrm{i}=1}^{\mathrm{N}}\left(\mathrm{X}_{\text {exp,i }}-\mathrm{X}_{\text {predi }}\right)^{2}\right]^{1 / 2} \\
\text { Teste } \mathrm{F}=\frac{\sum \overline{\mathrm{X}}_{\text {pred }}^{2}}{\sum \overline{\mathrm{X}}_{\text {resid }}^{2}}
\end{gathered}
$$

Em que: $X_{\text {exp,i é a umidade obtida }}$ experimentalmente, $X_{\text {pred ,i é a umidade }}$ predita pelo modelo e $\mathrm{N}$ é o número de experimentos.

Para a avaliação da variação da umidade de equilíbrio $\left(X_{e}\right)$ com a temperatura e a atividade de água $\left(\mathrm{a}_{\mathrm{w}}\right)$, para as sementes de ambas as variedades, foram determinadas as superfícies de resposta.

\subsection{CALOR DE SORÇÃO}

O calor líquido de sorção, conhecido também como entalpia diferencial $\left(\Delta \mathrm{h}_{\mathrm{st}}\right)$, foi determinado a partir da Equação de Clausius-Clayperon para cada umidade de equilíbrio, conforme mostra a Equação 4.

$$
\frac{\partial \ln \left(\mathrm{a}_{\mathrm{w}}\right)}{\partial \mathrm{T}}=\frac{\Delta \mathrm{h}_{\mathrm{st}}}{\mathrm{RT}_{\mathrm{a}}}
$$

Em que: $a_{w}$ é a atividade de água, $\mathrm{T}_{\mathrm{a}}$ é a temperatura absoluta $(\mathrm{K}), \Delta \mathrm{h}_{\mathrm{st}}$ é a entalpia diferencial $(\mathrm{kJ} / \mathrm{kg})$ e $\mathrm{R}$ a constante universal dos gases $(0,4619 \mathrm{~kJ} /$ kg.K).

Integrando a Equação 4 e assumindo que o calor líquido de sorção é independente da temperatura, obtém-se a 
Equação 5.

$$
\ln \left(a_{w}\right)=-\left(\frac{\Delta \mathrm{h}_{\mathrm{st}}}{\mathrm{R}}\right) \frac{1}{\mathrm{~T}_{\mathrm{a}}}+\mathrm{C}
$$

Em que: $\mathrm{C}$ é a constante do modelo.

O calor líquido de sorção foi determinado por regressão linear dos dados de $\ln \left(\mathrm{a}_{\mathrm{w}}\right)$ em função de $\left(1 / \mathrm{T}_{\mathrm{a}}\right)$ para os diferentes valores da umidade de equilíbrio (Equação 5). Este calor é o resultado numérico da multiplicação entre a inclinação das retas obtidas (para cada umidade de equilíbrio) e a constante universal dos gases.

$A$ atividade de água $\left(a_{w}\right)$ foi estimada pelo modelo de Henderson (1952), para as temperaturas de $25^{\circ} \mathrm{C}$, $35^{\circ} \mathrm{C}$ e $50^{\circ} \mathrm{C}$ e para umidades de equilíbrio de 2 a $15 \%$ (b.s.).

$\mathrm{O}$ calor integral de sorção $\left(\mathrm{Q}_{\mathrm{st}}\right)$ foi obtido adicionando-se aos valores de calor líquido de sorção, o valor do calor latente de vaporização da água livre, conforme mostra a Equação 6.

$$
\mathrm{Q}_{\mathrm{st}}=\Delta \mathrm{h}_{\mathrm{st}}+\mathrm{L}
$$

Em que $Q_{\text {st }}$ é o calor integral de sorção $(\mathrm{kJ} / \mathrm{kg})$, L é o calor latente de vaporização da água livre $(\mathrm{kJ} / \mathrm{kg})$.

\section{RESULTADOS E DISCUSSÃO}

\subsection{ISOTERMAS DE EQUILÍBRIO}

As isotermas de equilíbrio foram realizadas nas temperaturas de 25,35 e $50^{\circ} \mathrm{C}$, para as sementes de uva das variedades Cabernet Sauvignon e Bordô, sendo suas curvas apresentadas nas Figuras 2 e 3.

Nota-se pelas Figuras 2 e 3, que as curvas obtidas apresentaram um formato sigmoidal, característica de curvas do tipo II, de acordo com a classificação de Brunauer et al. (1938), que considera o formato do equilíbrio higroscópico padrão para produtos agrícolas.

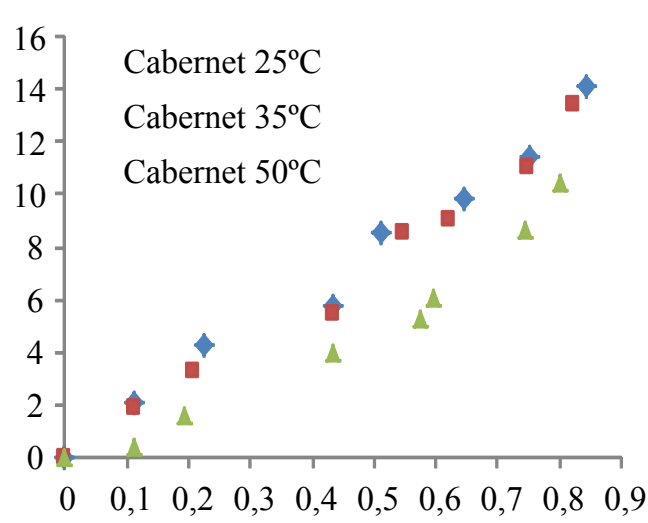

Atividade de Água (aw)

Figura 2. Isotermas de equilíbrio nas temperaturas de 25,35 e $50^{\circ} \mathrm{C}$ para sementes de Cabernet Sauvignon

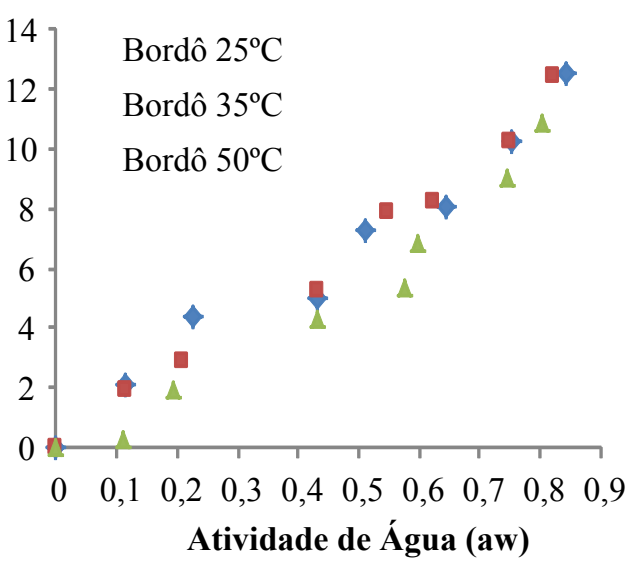

Figura 3. Isotermas de equilíbrio nas temperaturas de 25,35 e $50^{\circ} \mathrm{C}$ para sementes de Bordô

Pelas Figuras 2 e 3 foi verificado, também, que o aumento da temperatura de 25 para $35^{\circ} \mathrm{C}$ não apresentou influência na umidade de equilíbrio para as duas variedades estudadas. Já para a temperatura de $50^{\circ} \mathrm{C}$, a umidade de equilíbrio foi menor se comparada às outras temperaturas estudadas para ambas as variedades, mas podendo ser observada em maior proporção, nas sementes de Cabernet Sauvignon. Segundo Ascheri et al. (2005), em uma atividade de água constante, o aumento da temperatura promove redução do teor de água de equilíbrio, seguindo a tendência da maioria dos produtos agrícolas. Brooker et al. (1992) observaram este mesmo comportamento para o milho, sendo que 
um aumento na temperatura, com uma determinada umidade relativa constante, diminuiu o conteúdo de umidade de equilíbrio.

Com as isotermas de equilíbrio pode-se verificar que após o processo de secagem das sementes de uva, deve-se ter uma preocupação com o armazenamento das mesmas para que não haja uma absorção demasiada de umidade. Segundo as isotermas de equilíbrio das Figuras 2 e 3, para que as sementes permaneçam com um conteúdo de umidade menor que $10 \%$ (b.s.), a umidade relativa do ambiente de armazenamento não poderá ser maior que $75 \%$.

Para a atividade de água $\left(\mathrm{a}_{\mathrm{w}}\right)$ de aproximadamente 0,8 , a umidade de equilíbrio para sementes de Cabernet Sauvignon ficou em torno de $14 \%$ e para as sementes de Bordô, a umidade de equilíbrio ficou em torno de $12 \%$, para as temperaturas de 25 e $35^{\circ} \mathrm{C}$. Estes valores encontrados estão próximos às umidades de equilíbrio encontradas por ASAE (1991) para trigo, soja, arroz e amendoim. Já para a temperatura de $50^{\circ} \mathrm{C}$ e atividade de água $\left(\mathrm{a}_{\mathrm{w}}\right)$ de aproximadamente 0,8 , a umidade de equilíbrio para as duas variedades de sementes de uva avaliadas, ficou em torno de $10 \%$.

Nas Figuras 4 a 9, estão apresentados os ajustes realizados com os modelos de Henderson (1952), Henderson Modificado (1968), Motta Lima (1999), BET (1938), Sabbah (2001) e Oswin (1946), nas temperaturas de 25, 35 e $50^{\circ} \mathrm{C}$, para as sementes de Cabernet Sauvignon e Bordô.

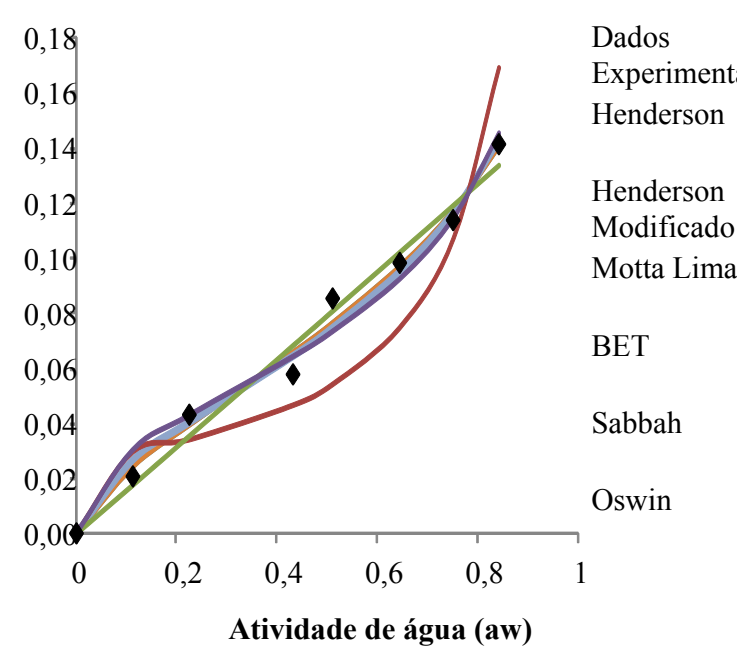

Figura 4. Ajuste dos modelos matemáticos aos dados experimentais das isotermas de equilíbrio na temperatura de $25^{\circ} \mathrm{C}$ para sementes de Cabernet Sauvignon

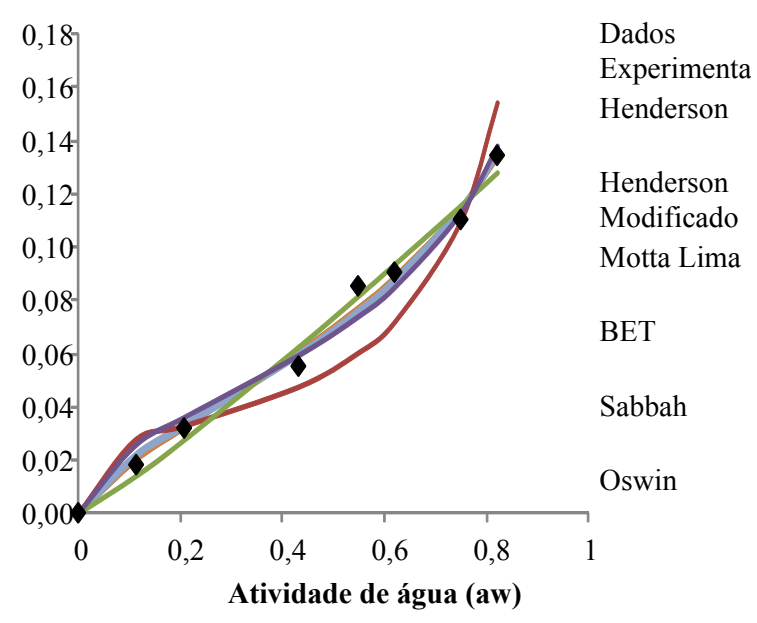

Figura 5. Ajuste dos modelos matemáticos aos dados experimentais das isotermas de equilíbrio na temperatura de $35^{\circ} \mathrm{C}$ para sementes de Cabernet Sauvignon 


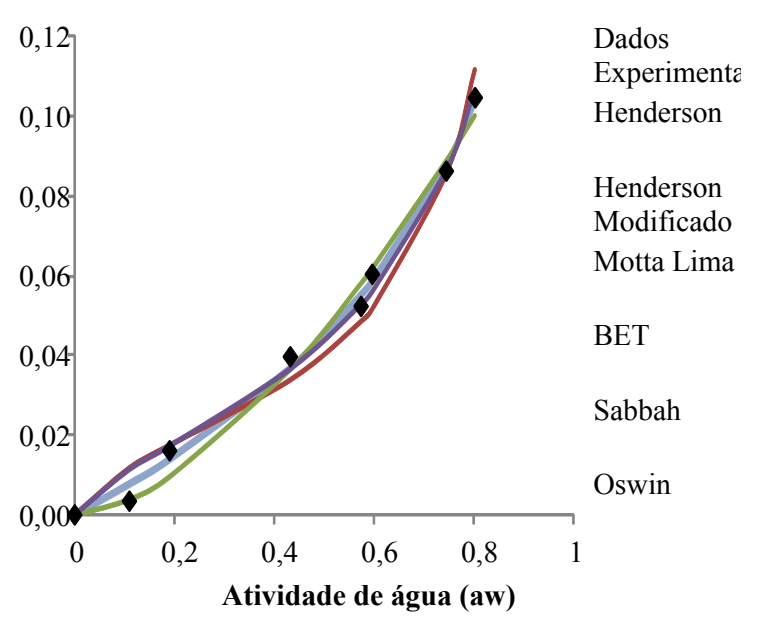

Figura 6. Ajuste dos modelos matemáticos aos dados experimentais das isotermas de equilíbrio na temperatura de $50^{\circ} \mathrm{C}$ para sementes de Cabernet Sauvignon

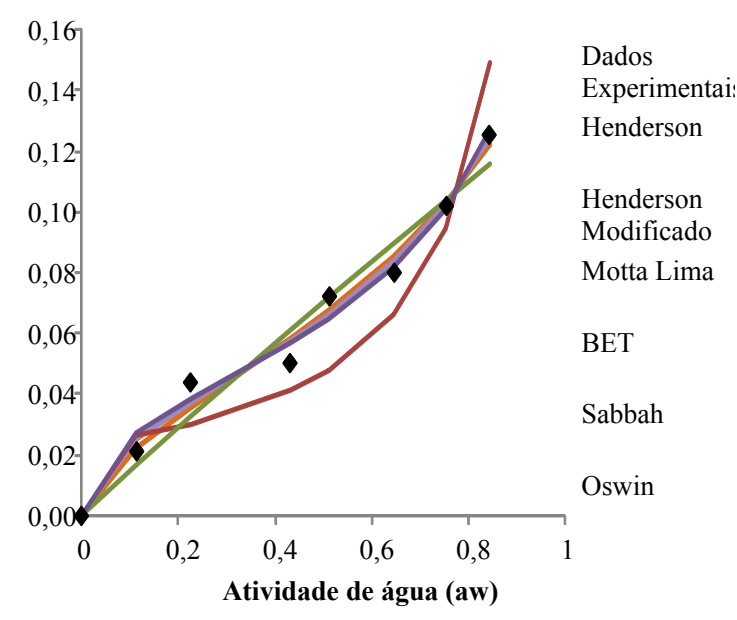

Figura 7. Ajuste dos modelos matemáticos aos dados experimentais das isotermas de equilíbrio na temperatura de $25^{\circ} \mathrm{C}$ para sementes de Bordô

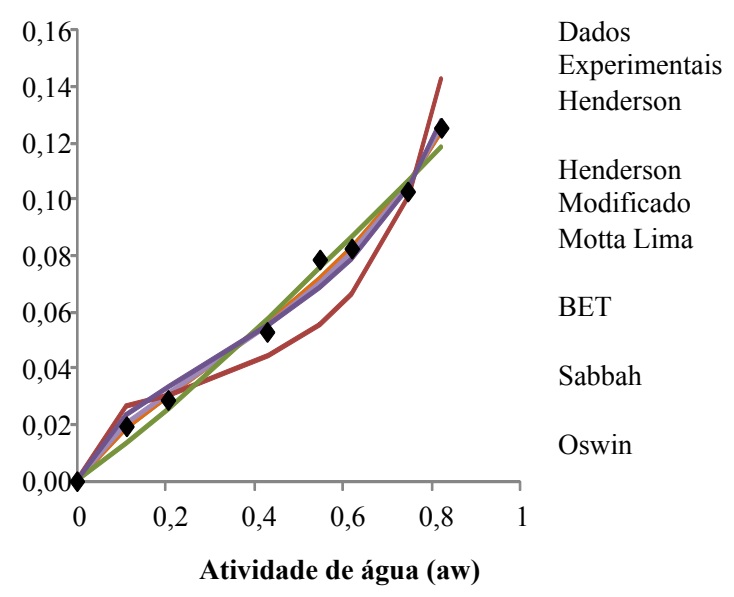

Figura 8. Ajuste dos modelos matemáticos aos dados experimentais das isotermas de equilíbrio na temperatura de ENGEVISTA, V. 17, n. 1, p. 44-58, Março 2015 $35^{\circ} \mathrm{C}$ para sementes de Bordô

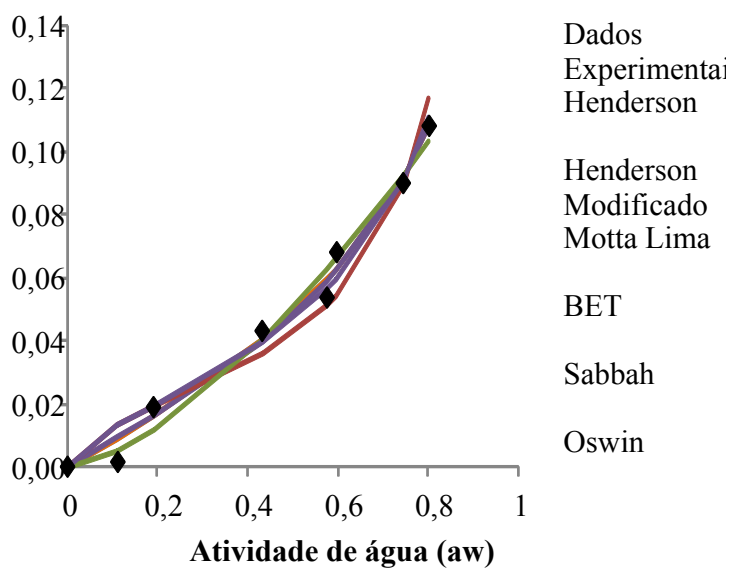

Figura 9. Ajuste dos modelos matemáticos aos dados experimentais das isotermas de equilíbrio na temperatura de $50^{\circ} \mathrm{C}$ para sementes de Bordô

Observando as Figuras 4 a 9, verifica-se, também, que o modelo de BET (1938) não apresentou um bom ajuste aos dados experimentais, principalmente para as menores temperaturas de secagem. Entretanto, somente observando as curvas, não foi possível determinar quais modelos melhor representaram os dados experimentais.

Desta forma, a escolha do melhor modelo foi feita com base nos maiores valores do coeficiente de determinação $\left(\mathrm{R}^{2}\right)$ e do Teste $\mathrm{F}$ e nos menores valores do RM, apresentados nas Tabelas 3 e 4.

A partir dos resultados apresentados na Tabela 3, os modelos que melhor se ajustaram às isotermas de equilíbrio experimentais para as sementes de Cabernet Sauvignon foram os modelos de Henderson e Henderson Modificado, para todas as temperaturas estudadas. $\mathrm{Na}$ temperatura de $25^{\circ} \mathrm{C}$, ambos os modelos obtiveram um $\mathrm{R}^{2}$ de 0,9890, um RM de $6,30.10^{-5}$ e um valor de 310,89 para o Teste F. Para a temperatura de $35^{\circ} \mathrm{C}$, os melhores modelos obtiveram um $\mathrm{R}^{2}$ de 0,9924, um RM de 4,13.10-5 e um valor de Teste $F$ de 427,27. Já na temperatura de $50^{\circ} \mathrm{C}$, estes modelos obtiveram um $\mathrm{R}^{2}$ de 0,9955 , um $\mathrm{RM}$ de $1,62.10^{-5}$ e um valor de Teste $\mathrm{F}$ de 581,09. 
Tabela 3. Valores de $\mathrm{R}^{2}$, RM e Teste F, para os ajustes dos modelos aos dados experimentais de isotermas de equilíbrio para sementes de Cabernet Sauvignon

\begin{tabular}{c|c|c|c|c|c|c|c|c|c}
\hline \multirow{2}{*}{ Modelo } & \multicolumn{4}{|c|}{$\mathbf{R}^{\mathbf{2}}$} & \multicolumn{3}{c|}{$\mathbf{R M}$} & \multicolumn{3}{c}{ Teste $\mathbf{~}$} \\
\cline { 2 - 10 } & $\mathbf{2 5}^{\circ} \mathbf{C}$ & $\mathbf{3 5}^{\circ} \mathbf{C}$ & $\mathbf{5 0}^{\circ} \mathbf{C}$ & $\mathbf{2 5}^{\circ} \mathbf{C}$ & $\mathbf{3 5}^{\circ} \mathbf{C}$ & $\mathbf{5 0}^{\circ} \mathbf{C}$ & $\mathbf{2 5}^{\circ} \mathbf{C}$ & $\mathbf{3 5}^{\circ} \mathbf{C}$ & $\mathbf{5 0}^{\circ} \mathbf{C}$ \\
\hline Henderson & 0,9890 & 0,9924 & 0,9955 & $6,30.10^{-5}$ & $4,13.10^{-5}$ & $1,62.10^{-5}$ & 310,89 & 427,27 & 581,09 \\
\hline $\begin{array}{c}\text { Henderson } \\
\text { Modificado }\end{array}$ & 0,9890 & 0,9924 & 0,9955 & $6,30.10^{-5}$ & $4,13.10^{-5}$ & $1,62.10^{-5}$ & 310,89 & 427,27 & 581,09 \\
\hline $\begin{array}{c}\text { Motta } \\
\text { Lima }\end{array}$ & 0,9864 & 0,9904 & 0,9954 & $7,81.10^{-5}$ & $5,20.10^{-5}$ & $1,63.10^{-5}$ & 250,44 & 339,32 & 578,25 \\
\hline BET & 0,8384 & 0,9017 & 0,9756 & $9,30.10^{-4}$ & $5,38.10^{-4}$ & $8,77.10^{-5}$ & 20,13 & 31,94 & 106,57 \\
\hline Sabbah & 0,9730 & 0,9882 & 0,9891 & $1,02.10^{-4}$ & $6,44.10^{-5}$ & $3,90.10^{-5}$ & 192,19 & 273,99 & 240,55 \\
\hline Oswin & 0,9796 & 0,9828 & 0,9900 & $1,17.10^{-4}$ & $9,39.10^{-5}$ & $3,58.10^{-5}$ & 166,64 & 187,65 & 262,33 \\
\hline
\end{tabular}

Tabela 4. Valores de $\mathrm{R}^{2}$, RM e Teste F, para os ajustes dos modelos aos dados experimentais de isotermas de equilíbrio para sementes de Bordô

\begin{tabular}{c|c|c|c|c|c|c|c|c|c}
\hline \multirow{2}{*}{ Modelo } & \multicolumn{5}{|c|}{$\mathbf{R}^{\mathbf{2}}$} & \multicolumn{3}{c|}{$\mathbf{R M}$} & \multicolumn{3}{c}{ Teste $\mathbf{F}$} \\
\cline { 2 - 10 } & $\mathbf{2 5}^{\circ} \mathbf{C}$ & $\mathbf{3 5}^{\circ} \mathbf{C}$ & $\mathbf{5 0}^{\circ} \mathbf{C}$ & $\mathbf{2 5}^{\circ} \mathbf{C}$ & $\mathbf{3 5}^{\circ} \mathbf{C}$ & $\mathbf{5 0}^{\circ} \mathbf{C}$ & $\mathbf{2 5}^{\circ} \mathbf{C}$ & $\mathbf{3 5}^{\circ} \mathbf{C}$ & $\mathbf{5 0}^{\circ} \mathbf{C}$ \\
\hline Henderson & 0,9857 & 0,9941 & 0,9881 & $7,00.10^{-5}$ & $2,70.10^{-5}$ & $4,65.10^{-5}$ & 214,69 & 560,00 & 223,97 \\
\hline $\begin{array}{c}\text { Henderson } \\
\text { Modificado }\end{array}$ & 0,9857 & 0,9941 & 0,9881 & $7,00.10^{-5}$ & $2,70.10^{-5}$ & $4,65.10^{-5}$ & 214,69 & 560,00 & 223,97 \\
\hline $\begin{array}{c}\text { Motta } \\
\text { Lima }\end{array}$ & 0,9835 & 0,9929 & 0,9878 & $7,30.10^{-5}$ & $3,27.10^{-5}$ & $4,74.10^{-5}$ & 208,40 & 462,61 & 219,65 \\
\hline BET & 0,8571 & 0,9089 & 0,9611 & $6,09.10^{-4}$ & $4,25.10^{-4}$ & $1,53.10^{-4}$ & 23,84 & 34,77 & 67,67 \\
\hline Sabbah & 0,9625 & 0,9872 & 0,9833 & $1,59.10^{-4}$ & $5,92.10^{-5}$ & $6,52.10^{-5}$ & 93,91 & 255,51 & 159,65 \\
\hline Oswin & 0,9851 & 0,9867 & 0,9809 & $6,06.10^{-5}$ & $6,12.10^{-5}$ & $7,49.10^{-5}$ & 208,95 & 247,11 & 138,89 \\
\hline
\end{tabular}

A partir dos resultados da Tabela 4, nota-se que os modelos que melhor se ajustaram às isotermas de equilíbrio experimentais para as sementes de Bordô foram os modelos de Henderson e Henderson Modificado, para todas as temperaturas estudadas. Na temperatura de $25^{\circ} \mathrm{C}$, ambos os modelos obtiveram um $\mathrm{R}^{2}$ de 0,9857, um RM de 7,00.105 e um valor de 214,69 para o Teste F. Para a temperatura de $35^{\circ} \mathrm{C}$, os melhores modelos obtiveram um $\mathrm{R}^{2}$ de 0,9941 , um RM de $2,70.10^{-5}$ e um valor de Teste $\mathrm{F}$ de 560,00 . Já na temperatura de $50^{\circ} \mathrm{C}$, estes modelos obtiveram um $\mathrm{R}^{2}$ de 0,9881 , um RM de 4,65.10-5 e um valor de Teste F de 223,97.

Comparando os resultados obtidos com a literatura, Corrêa et al. (2006) estudaram o equilíbrio higroscópico de milheto, alpiste e painço, obtendo também, bons ajustes com os modelos de Henderson Modificado, para milheto, e Oswin, para alpiste e painço. Mesquita et al. (2010) verificaram que o modelo de Henderson Modificado foi o que melhor se ajustou aos dados de umidade de equilíbrio para as sementes de óleocopaíba. Segundo Oliveira et al. (2005), a equação de Henderson (1952) é uma das mais utilizadas para a predição de isotermas.

Os parâmetros obtidos por regressão não linear dos modelos de melhor ajuste estão apresentados nas Tabelas 5 e 6 para as sementes de Cabernet Sauvignon e Bordô, respectivamente.

Tabela 5. Parâmetros dos modelos de melhor ajuste para as isotermas de 
equilíbrio das sementes de Cabernet

Sauvignon nas temperaturas de 25, 35 e

\begin{tabular}{c|c|c|c|c}
\multicolumn{5}{c}{$50{ }^{\circ} \mathrm{C}$} \\
\hline Modelo & $\mathbf{T}\left({ }^{\circ} \mathbf{C}\right)$ & $\mathbf{B}$ & $\mathbf{C}$ & $\mathbf{D}$ \\
\hline \multirow{4}{*}{ Henderson } & 25 & 0,6401 & & 0,7447 \\
\cline { 2 - 5 } & 35 & 0,7093 & & 1,1315 \\
\cline { 2 - 5 } & 50 & 0,9838 & & 3,0280 \\
\hline \multirow{3}{*}{$\begin{array}{c}\text { Henderson } \\
\text { Modificado }\end{array}$} & 25 & 0,9058 & 1,5622 & 1,5294 \\
\cline { 2 - 5 } & 35 & 0,8636 & 1,4097 & 0,8198 \\
\cline { 2 - 5 } & 50 & 0,1801 & 1,0164 & 0,3231 \\
\hline
\end{tabular}

Tabela 6. Parâmetros dos modelos de melhor ajuste para as isotermas de equilíbrio das sementes de Bordô nas temperaturas de 25,35 e $50{ }^{\circ} \mathrm{C}$

\begin{tabular}{c|c|c|c|c}
\hline Modelo & $\mathbf{T}\left({ }^{\circ} \mathbf{C}\right)$ & $\mathbf{B}$ & $\mathbf{C}$ & $\mathbf{D}$ \\
\hline \multirow{4}{*}{ Henderson } & 25 & 0,6226 & & 0,6187 \\
\cline { 2 - 5 } & 35 & 0,7049 & & 1,0327 \\
\cline { 2 - 5 } & 50 & 0,9838 & & 3,0280 \\
\hline \multirow{3}{*}{$\begin{array}{c}\text { Henderson } \\
\text { Modificado }\end{array}$} & 25 & 1,0628 & 1,6061 & 2,0738 \\
\cline { 2 - 5 } & 35 & 0,3521 & 1,4185 & 0,9458 \\
\cline { 2 - 5 } & 50 & 0,1801 & 1,0164 & 0,3231 \\
\hline
\end{tabular}

\subsection{ANÁLISE ESTATÍSTICA}

Para as isotermas de equilíbrio das sementes de uva das variedades Cabernet Sauvignon e Bordô, os modelos ajustados para a resposta umidade de equilíbrio são dados pelas Equações 7 e 8 , respectivamente.

$$
\begin{aligned}
& X e=-3,2+14 . X_{T}+6,08 X_{T}{ }^{2}+ \\
& +0,24 \cdot X_{a w}-0,004 X_{a w}{ }^{2}-0,12 X_{T} \cdot X_{a w} \\
& X e=-21+8,9 X_{T}+5,9 X_{T}^{2}+0,2 X_{a w}{ }^{+} \\
& -0,003 X_{a w}{ }^{2}-0,008 X_{T} X_{a w}
\end{aligned}
$$

As Figuras 10 e 11 apresentam as superfícies de resposta da umidade de equilíbrio em função da atividade de água e da temperatura para as sementes de uva das variedades Cabernet Sauvignon e Bordô, respectivamente.

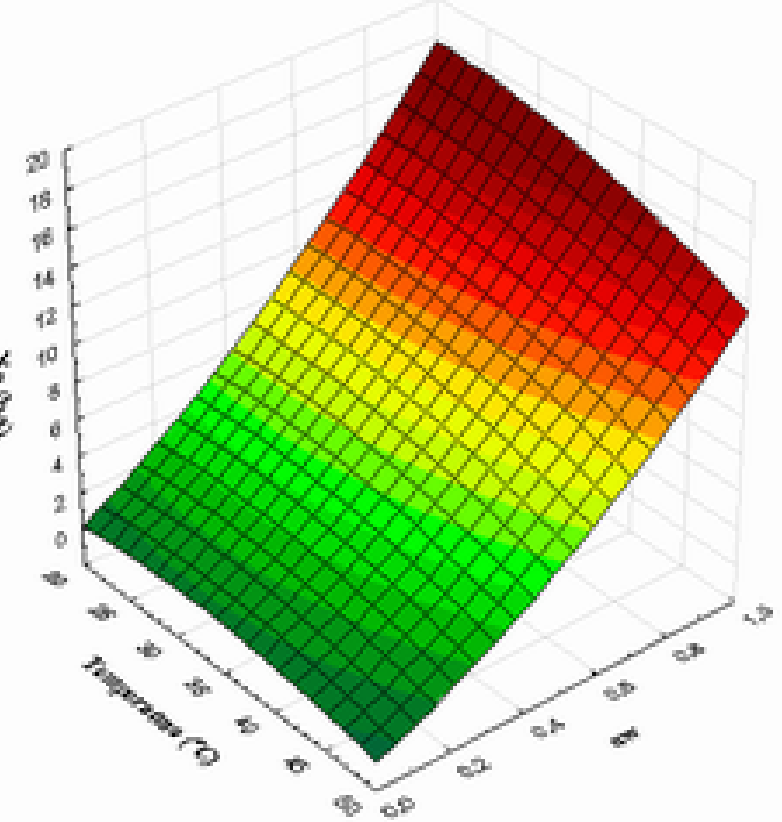

Figura 10. Superfície de resposta obtida para as sementes de Cabernet Sauvignon

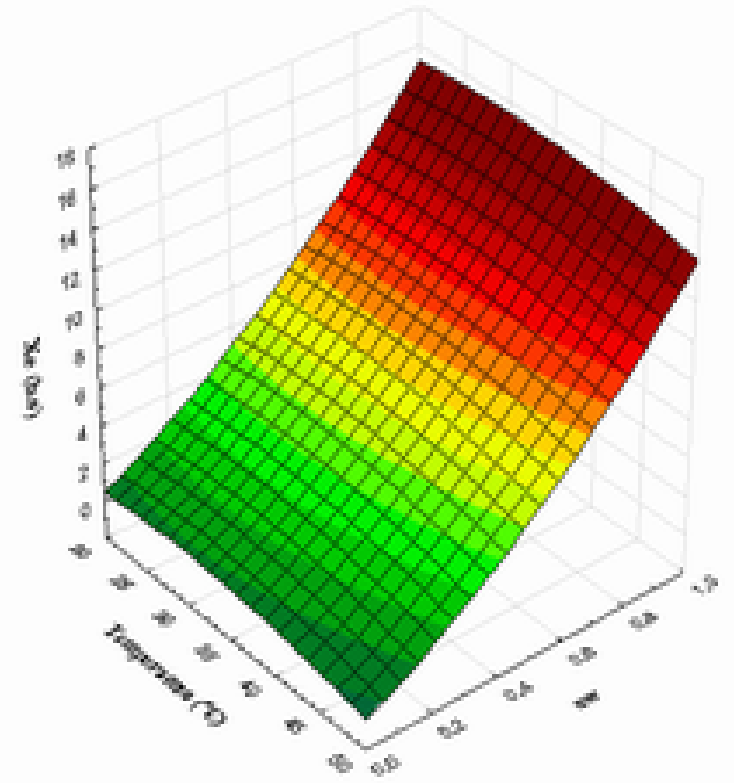

Figura 11. Superfície de resposta obtida para as sementes de Bordô

Verifica-se pelas Figuras $10 \mathrm{e}$ 11, que a faixa de temperatura estudada não apresentou influência significativa na umidade de equilíbrio das sementes. Comportamento diferente pode ser observado com relação à atividade de água, pois seu aumento também provocou um aumento da umidade de equilíbrio das sementes de ambas as variedades.

Nas Figuras 10 e 11 também se pode observar que, para um valor constante de atividade de água, o aumento da temperatura promoveu a 
redução do teor de umidade de equilíbrio, seguindo a tendência da maioria dos produtos agrícolas (Ascheri et al., 2005).

As Figuras 12 e 13 apresentam os gráficos de resíduos obtidos para ambas as variedades de semente, sendo que os valores dos resíduos foram determinados pela diferença entre os valores obtidos experimentalmente e os obtidos pelo modelo.

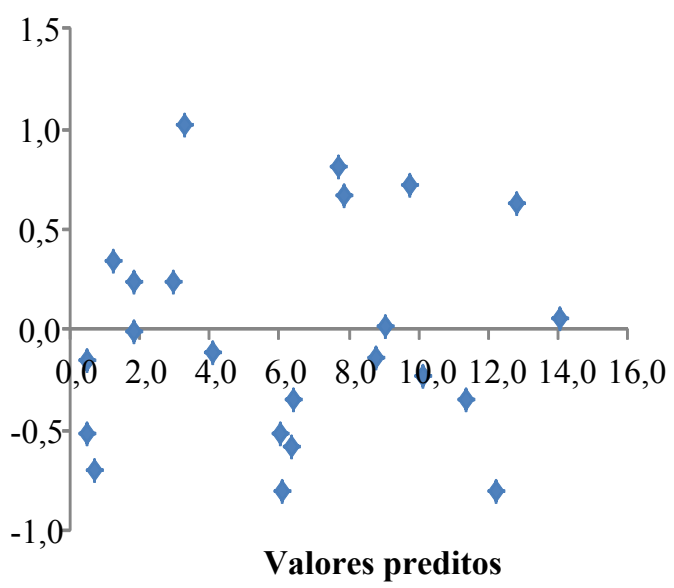

Figura 12. Gráfico de resíduos obtido para as sementes de uva da variedade Cabernet Sauvignon

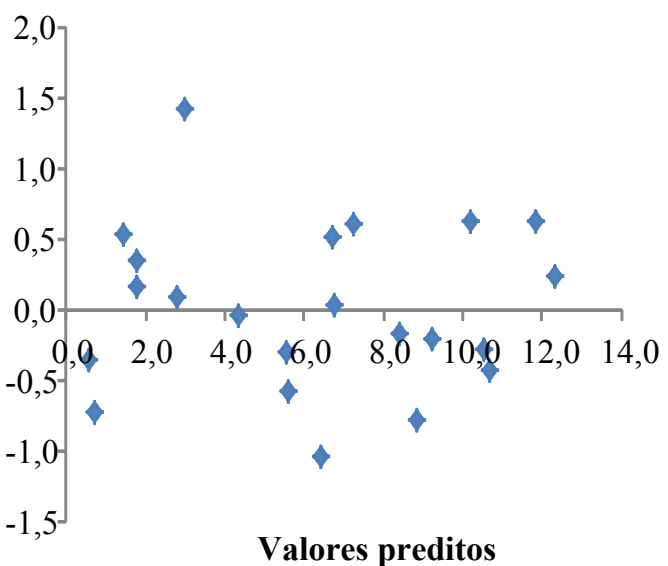

Figura 13. Gráfico de resíduos obtido para as sementes de uva da variedade Bordô

Nas Figuras 12 e 13 pode-se observar que os resíduos apresentaram uma distribuição aleatória em torno do zero, não apresentando tendência específica dos pontos. Este comportamento indica que os modelos apresentados nas Equações 7 e 8 tiveram um bom ajuste aos dados experimentais de isotermas de equilíbrio, com um $\mathrm{R}^{2}$ de 0,9927 e 0,9894 para as sementes de Cabernet Sauvignon e Bordô, respectivamente.

\subsection{CALOR DE SORÇÃO}

A partir do modelo de Henderson (1952) e dos valores de seus parâmetros, foram obtidos os valores da atividade de água para cada respectivo valor da umidade de equilíbrio, em cada temperatura estudada. Os resultados desses cálculos estão apresentados nas Tabelas 7 e 8, para as sementes de uva das variedades Cabernet Sauvignon e Bordô, respectivamente.

Tabela 7. Valores de atividade de água estimados pelo modelo de Henderson (1952), para as sementes de Cabernet

\begin{tabular}{|c|c|c|c|}
\hline \multicolumn{4}{|c|}{ Sauvignon } \\
\hline \multirow{2}{*}{$\begin{array}{c}\mathrm{Xe} \\
(\% \text { b.s })\end{array}$} & \multicolumn{3}{|c|}{ Temperaturas $\left({ }^{\circ} \mathrm{C}\right)$} \\
\hline & 25 & 35 & 50 \\
\hline 2 & $\begin{array}{c}0,084 \\
1\end{array}$ & 0,1157 & 0,1611 \\
\hline 5 & $\begin{array}{c}0,307 \\
6\end{array}$ & 0,3499 & 0,5378 \\
\hline 7 & $\begin{array}{c}0,463 \\
0\end{array}$ & 0,4995 & 0,6626 \\
\hline 9 & $\begin{array}{c}0,601 \\
8 \\
\end{array}$ & 0,6271 & 0,7540 \\
\hline 11 & $\begin{array}{c}0,716 \\
3\end{array}$ & 0,7299 & 0,8209 \\
\hline 13 & $\begin{array}{c}0,805 \\
2\end{array}$ & 0,8092 & 0,8697 \\
\hline 15 & $\begin{array}{c}0,870 \\
7\end{array}$ & 0,8683 & 0,9053 \\
\hline
\end{tabular}

Tabela 8. Valores de atividade de água estimados pelo modelo de Henderson (1952), para as sementes de Bordô

\begin{tabular}{c|c|c|c}
\hline \multirow{2}{*}{$\begin{array}{c}\text { Xe } \\
\text { \% b.s })\end{array}$} & \multicolumn{3}{|c}{ Temperaturas $\left({ }^{\mathbf{o}} \mathbf{C}\right)$} \\
\cline { 2 - 4 } & $\mathbf{2 5}$ & $\mathbf{3 5}$ & $\mathbf{5 0}$ \\
\hline & 0,096 & & \\
2 & 0 & 0,1219 & 0,2622 \\
\hline & 0,355 & & \\
5 & 8 & 0,3794 & 0,5378 \\
\hline & 0,529 & & \\
7 & 9 & 0,5365 & 0,6626 \\
\hline & 0,677 & & \\
9 & 1 & 0,6665 & 0,7540 \\
\hline & 0,789 & & \\
11 & 9 & 0,7677 & 0,8209 \\
\hline & 0,870 & & \\
13 & 0 & 0,8428 & 0,8697 \\
\hline
\end{tabular}




\section{\begin{tabular}{l|c|l|l} 
& 0,923 & & \\
15 & 3 & 0,8964 & 0,9053 \\
\hline
\end{tabular}}

Pelas Tabelas 7 e 8 , verificase que os maiores valores da atividade de água estimados pelo modelo de Henderson foram obtidos na menor temperatura de secagem $\left(25^{\circ} \mathrm{C}\right)$ e na maior umidade de equilíbrio.

Nas Figuras 14 e 15 estão apresentadas as curvas ajustadas para $\ln \left(a_{\mathrm{w}}\right)$ em função de $(1 / \mathrm{T})$, para as sementes de uva das variedades Cabernet Sauvignon e Bordô, respectivamente.

Figura 14. Curvas de $\ln \left(\mathrm{a}_{\mathrm{w}}\right)$ para valores específicos de umidade de equilíbrio (\% b.s.) para as sementes de uva da variedade Cabernet Sauvignon indicando que a equação de Henderson Modificado (1952) é adequada para a determinação do calor de sorção das sementes nas condições estudadas.

Os valores do calor integral de sorção das sementes de uva variaram de $2329 \mathrm{~kJ} / \mathrm{kg}$ a $3403 \mathrm{~kJ} / \mathrm{kg}$ e de 2225 a $4077 \mathrm{~kJ} / \mathrm{kg}$, para as sementes de uva das variedades Cabernet Sauvignon e Bordô, respectivamente. Comparando-se os valores, nota-se que o calor integral de sorção para as sementes de uva da variedade Bordô foi maior que para as sementes da variedade Carbernet Sauvignon. Este resultado indica que as sementes da variedade Bordô apresentam uma maior resistência para perder água e, consequentemente, torna-se necessária mais energia para a secagem desta variedade.

De acordo com Teixeira et al. (2012), um produto exposto a uma condição em que há perda de umidade, em um primeiro momento, a energia fornecida para a retirada da água é menor do que aquela fornecida ao final do processo devido ao decaimento da disponibilidade de água.

Este fenômeno se deve ao fato de que quando o material apresenta menor umidade, a energia de interação entre a primeira camada de sorção do sólido e as moléculas de água é maior do que a energia que mantém as moléculas unidas nas multicamadas. Assim, a quantidade de energia necessária para remover a água do material durante a secagem aumentará com a redução da umidade (Johann et al., 2010).

As Figuras 16 e 17 apresentam o comportamento do calor integral de sorção para ambas as variedades de semente de uva.
Figura 15. Curvas de $\ln \left(a_{\mathrm{w}}\right)$ para valores específicos de umidade de equilíbrio (\% b.s.) para as sementes de uva da variedade Bordô

Observa-se nas Figuras 14 e 15 uma relação linear entre $\ln \left(\mathrm{a}_{\mathrm{w}}\right)$ e $1 / \mathrm{T}$, 
Figura 16. Valores experimentais e estimados do calor integral de sorção para as sementes de Cabernet Sauvignon
Figura 17. Valores experimentais e estimados do calor integral de sorção para as sementes de Bordô

Observando as Figuras 16 e 17 verifica-se que, com o aumento da umidade de equilíbrio, o calor de sorção aproxima-se do calor de vaporização da água na mesma temperatura $(2256 \mathrm{~kJ} /$ $\mathrm{kg}$ ). Isto ocorre porque quanto maior a quantidade de água presente no material, maior é o enfraquecimento da energia de ligação entre as moléculas, aproximandose da energia de ligação das moléculas de água em estado líquido.

\section{CONCLUSÃO}

Analisando os resultados foi possível verificar que, para que as sementes de ambas as variedades permaneçam com uma umidade de $10 \%$ (b.s.) durante o armazenamento, a umidade relativa do ambiente não poderá ser maior que $75 \%$.

Os modelos analisados apresentaram bons ajustes aos dados experimentais (com coeficientes de determinação maiores que 0,9 ), exceto para o modelo de BET que obteve os valores mais baixos do $R^{2}$ e Teste $F$ e os maiores valores do RM para todas as condições estudadas.

Em relação ao calor integral de sorção, observou-se que à medida que a umidade de equilíbrio diminui, ocorre, também, a diminuição da umidade livre, e consequentemente, a energia necessária para sua evaporação aumenta. Os valores encontrados ficaram entre $2329 \mathrm{~kJ} /$ $\mathrm{kg}$ e $3403 \mathrm{~kJ} / \mathrm{kg}$, para as sementes da variedade Cabernet Sauvignon e entre 2225 e $4077 \mathrm{~kJ} / \mathrm{kg}$ para as sementes da variedade Bordô.

\section{REFERÊNCIAS}

American Society of Agricultural Engineers - ASAE. Standard: D245.4: Moisture Relationship of Grains, 1991.

American Society of agricultural Engineers - ASAE. Standards engineering practices data. St. joseph, MI, 1993. 784p.

ASCHERI, D. P. R.; ANDRADE, C. T; CARVALHO, C. W. P; ASCHERI, J. L. R. Isotermas de adsorción de agua y estabilidad de harinas extruídas de amaranto, arroz y maiz: Estudio comparativo. Alimentaria, Madrid, v. 42, n. 363 , p. 100-107, 2005.

BARROZO, M. A. S.; OLIVEIRA, D. T.; SANCINETI, G. P.; RODRIGUES, M. V. A study of the desorption isotherms of lentils. Brazilian Journal of Chemical Engineering, v. 17, p. 105-109, 2000.

BROOKER, D. B.; BAKKERARKEMA, F. W.; HALL, C. W. Grain equilibrium moisture content. In: Drying and storage of grains and oilseeds, New York, p.67-86, 1992. 
BRUNAUER, S.; EMMET, P. H.; TELLER, E. Adsorpition of gases in multimolecular layers. Journal American Chemical Sociaty, v. 60, n. 2, p. 309-319, 1938.

CHEN, C.; JAYAS, D. S. Evaluation of the GAB equation for the isotherms of agricultural products. Transactions of the ASAE, St. Joseph, v. 41, n. 6, p. 17551760, 1998.

CHETANA, R.; SRINIVASA, P. C.; REDDY, S. R. Y. Moisture sorption characteristics of milk burfi, an traditional Indian sweet, using sugar substitutes. European Food Research and Technology, v. 220, n. 2, p. 136-141, 2005.

CHIRIFE, J.; IGLESIAS, H. A. Equations for fitting water sorption isotherms of foods: Part 1 - a review. Journal of Food Technology, v. 13, p. 159-174, 1978.

CORRÊA, P. C.; JÚNIOR, P. C. A.; RIBEIRO, D. M.; SILVA, F. S. Equilíbrio hogroscópico de milheto, alpiste e painço: Obtenção e modelagem. Revista Brasileira de Engenharia Agrícola e Ambiental, v. 10, n. 1, p. 162$167,2006$.

DITCHFIELD, C. 2000. Estudo dos métodos para medida da atividade de água. Dissertação de Mestrado, Escola Politécnica da Universidade de São Paulo.

FIORENTIN, L. D.; MENON, B. T.; ALVES, J. A.; BARROS, S. T. D.; PEREIRA, N. C.; MÓDENES A. N. Determinação da cinética e das isotermas de secagem do bagaço da laranja. Acta Scientiarum. Technology, v. 32, n. 2 p. 147-152, 2010.

FOUST, A. S. WENZEL, L. A.; CLUMP, C. W. Principles of Unit Operations, 2nd Ed., John Wiley \& Sons Inc., 1982.
FREITAS, L. S. 2007. Desenvolvimento de procedimentos de extração do óleo de semente de uva e caracterização química dos compostos extraídos. Tese de Doutorado, Instituto de Química, Universidade Federal do Rio Grande do Sul.

HENDERSON, S. M. A basic concept of equilibrium moisture. Agricultural Engineering, v. 33, n. 1, p. 29-32, 1952.

JOHANN, G.; PEREIRA, N. C.; MOTTA LIMA, O. C.; SILVA, E. A. Determinação do calor de dessorção para materiais têxteis. Acta Scientiarum. Technology, v. 32, n. 1, p. 63-68, 2010.

LABUZA, T. P. Sorption phenomena in foods. Food Technology, v. 22, n. 3, p. 15-24, 1968.

MESQUITA, J. B.; ANDRADE, E. T. DE; CORRÊA, P. C. Modelos matemáticos e curvas de umidade de equilibrio de sementes de jacarandá da bahia, angico-vermelho e óleo-copaíba. CERNE, v. 7, n. 2, p. 12-21, 2001.

MIGUEL, L. Formulações com óleo de sementes de uva "Suvalan". Porto Alegre: Faculdade de Farmácia da UFRS, 1983.

MOTTA LIMA, O. C. 1999. Estudo sobre a secagem de folhas de celulose. Tese de Doutorado, Universidade Federal do Rio de Janeiro.

OSWIN, C. R. The kinetics of packing life. III. The isotherm. Journal of Chemistry Industrial, n.65, p.419-23, 1946.

OLIVEIRA, M. M.; CAMPOS, A, R. N.; GOMES, J. P.; SILVA, F. L. H. Isotermas de sorção do resíduo agroindustrial de casca do abacaxi (Ananas comosus L. Mer). Revista Brasileira de Engenharia Agrícola e Ambiental, v.9, n.4, p.565-569, 2005. 
PERRY, R. H.; CHILTON, C. H. Manual de Engenharia Química, 5 ed., Guanabara Dois, Rio de Janeiro, 1980.

ROBERTS, J. S; KIDD, D. R.; PADILLA-ZAKOUR, O. Drying kinetics of grape seeds. Journal of Food Engineering, v. 89, p. 460-465, 2008.

S'ENYI, J. M. Fruit Processing Waste Management. In: Hui, Y. H. Handbook of Fruits and Fruit Processing. 1st. ed. Blackwell Publishing, p. 171-186, 2006.

SOUSA, L. H. C. D. Estudo da secagem de materiais têxteis. Tese (Doutorado), Universidade Estadual de Maringá, 2003.

TEIXEIRA, L. P; ANDRADE, E. T.; SILVA, P. G. L. Determinação do equilibrio higroscópico e do calor isostérico da polpa e da casca do abacaxi (Ananas comosus). Engevista, v. 14, p. 172-184, 2012.

THOMPSON, T. L; PEART, R. M.; FOSTER, G. H. Mathematical simulation of corn drying - A new model. Transaction of the ASAE, St. Joseph, v. 11, n. 4, p. 582-586, 1968.

YOSHIDA, C. M. P. 1997. Cinética de secagem do milho superdoce. Dissertação de Mestrado, Universidade Estadual de Campinas. 